Military Technical College Kobry El-Kobbah, Cairo, Egypt

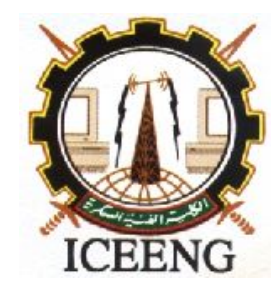

\author{
$9^{\text {th }}$ International Conference \\ on Electrical Engineering \\ ICEENG 2014
}

\title{
Two Axes Automatic Solar Panel Sun Tracking System For Supplying Power To Mine Detection Robot
}

\author{
by \\ Eng. Walid Mohamed mostafa \\ Department of Industrial Electronics Engineering \\ Faculty of Electronic Eng., Menoufia University \\ Prof. Dr. Eng. Adle Ezzat Elhenawy \\ Department of Electrical Electronic and Telecommunication \\ Engineering, Ain shams University \\ Prof. Dr. Eng. Magdy A. koutb \\ Department of Industrial Electronics Engineering \\ Faculty of Electronic Eng., Menoufia University
}

\section{$\underline{\text { Abstract: }}$}

This paper presents a two axes automatic solar panel tracking system installed on an automated mine detection robot to supply the robot with electrical energy. The tracking system is controlled using a microcontroller programmed with astronomical algorithm to keep the solar panel at right angle with the incident sunrays, in order to improve the performance and to maximize the output power of the solar panel delivered all over the day hours and during the robot's motion in the field. Tracking system mechanism is rotated around two axes, tracing the sun angles from the sunrise to the sunset. The new positioning tracking system includes one photovoltaic (PV) panel of 90-watt output power. A liquid capacitive inclinometer chip is used for measuring the tilt angle of the solar panel with respect to ground and robot's inclined angle within 0 to 90 degree range. Electronic compass issued for adjusting the azimuth solar angle direction for any movement of the robot on the ground. A PWM controlled DC-DC charge controller 
Boost converter is used along with the automatic tracking system for enhancing the solar panel's output power under variable insolation conditions. Control of the overall system has implemented using PIC 18F452 microcontroller circuit. Practical results show the superiority of the illustrated design.

\section{Keywords:}

Solar tracking system on robot and charge controller Boost converter

\section{Introduction:}

To insure a remote permanent green, unlimited and reliable energy sources to supply electrical power to the mine detection robot that performs searching, detecting, catching and working in a minefield. An effective and harmless energy source that assures this objective is the solar energy, especially because Egypt belongs to the global Sun Belt. Egypt is a country that has advantageous position for generation of solar energy; it enjoys 2900-3200 hours of sunshine annually with annual direct average normal energy density of $4000 \mathrm{kWh} / \mathrm{m} 2$, [1].

Therefore, to collect the solar energy, an important factor concerning the efficiency of photovoltaic system output energy is the angle of incidence of sunlight on the surface of the panel. In the case of fixed panels, sunlight hits the solar cells at an oblique angle for most of the day. Maximum of electric energy is achieved only when sunlight strikes the cells perpendicular to their surface at noon. It is simple to mount solar panels at a fixed tilt and azimuth direction and just leave them there on the robot's board assuming that the robot do not move or moving only straight ahead on a clear smoothing ground. Unfortunately, this is not true because the robot move according to a searching path that may be in a certain position makes the solar panel out of angles and do not face the sunrays. With different observation location and observation time, the sunlight direction is different; therefore, sun's position must be tested and located first if the sunlight needs to be tracked. There are four available methods in order to achieve this; (1) timing method, (2) coordinates method, (3) relative method of sunlight strength to solar panels and (4) relative method of sunlight strength to photo resistance for detecting sunlight strength. After comparing and screening the four methods, it is thought that timing method only is simpler, but the precision of control system is inferior due to season impact. Relative method of sunlight strength makes the utilization efficiency of solar energy not be optimal. Relative method of sunlight strength to photo resistance represents the simplest hardware circuit and good utilization efficiency of solar energy. So, it is frequently used, but in robots application and also in different insolation conditions, it will not be the optimum method, [2]. Coordinates method has the 
complexity of the control circuit though the precision of control system is superior, so it is the best method be applied in that mine detection robot behavior.

An automatic solar panel coordinates tracking system installed on movable robot provides vertical light falling on the panel surface that maximizes the total power efficiency delivered to the robot batteries. Changing of the angles of the sun across the sky influences the major parameters that affect the tracking algorithm. These parameters are the altitude and the azimuth angle. Altitude is the angular distance above the horizon and measured perpendicularly to the horizon. It has maximum value of $90^{\circ}$ at zenith, which is the point overhead. Azimuth is the angular distance measured along the horizon in degrees corresponding to the compass direction from which the sunlight is coming. At solar noon, the sun is always directly south in the northern hemisphere of the earth. Azimuth starts from exactly north, at 0 degree, and increases clockwise. The altitude is symbolized by ( ) starts from the horizon, while $(\mathrm{Z})$ symbolizes the azimuth angle, starts from the South Pole and travels clockwise. Figure 1 shows that the sun is higher in summer more than spring and winter, and the direction of looking into the sun is the south. So, photovoltaic panels are positioned at the true south direction facing the sun with tilt angle depends on the region at which it is located. In the north halve of the earth as in Egypt, the (PV) panels is directed towards the south with optimal elevation tilt angle for that region. The tilt angle varies seasonally because the sun is higher in the summer and lowers in winter.

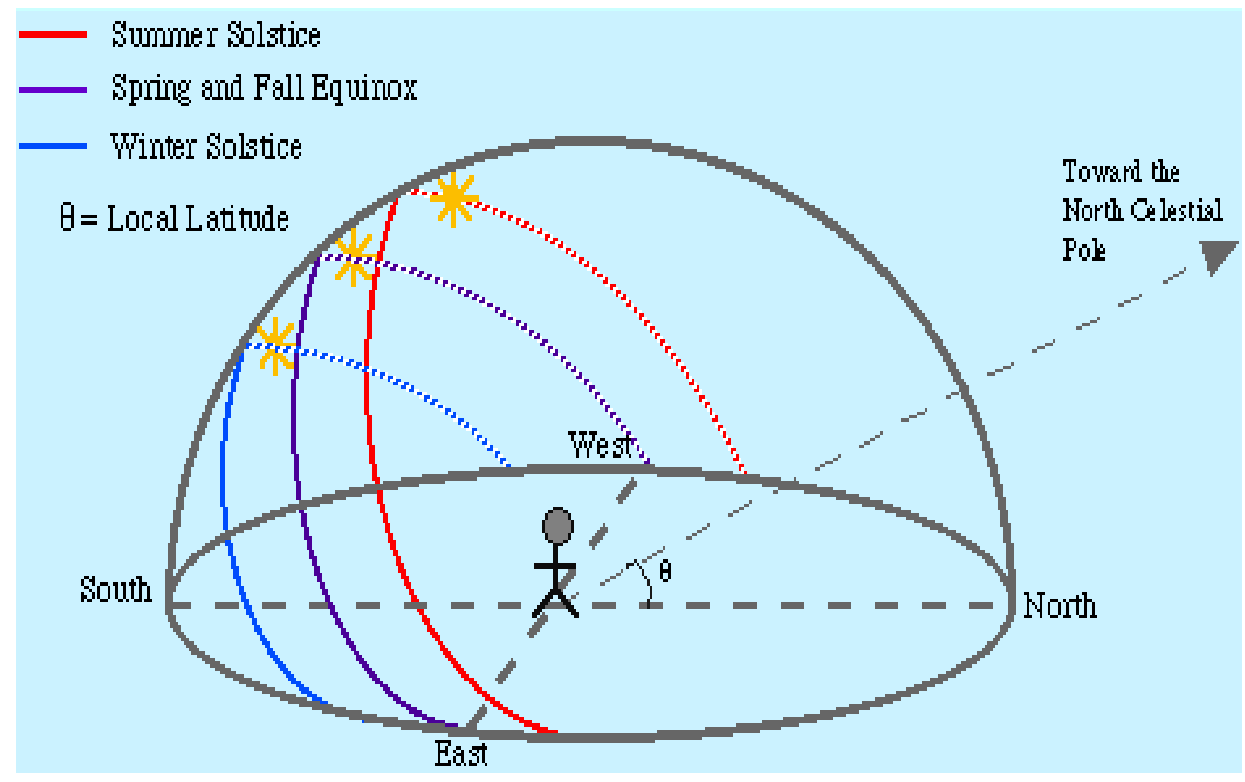

Figure (1) Sun path in the sky relative to the northern hemisphere and the seasons of the year . 
The sun's paths are different due to factors such as :

1) Location (local latitude) which in Egypt directed to south and the corresponding declination angle.

2) Rising and setting position based on time of the year and on the season (tilt angle).

3) Duration of day and night (from east to west).

To capture more energy during the year it is necessary to adjust the tilt of the panel according to the season.

Declination is the tilt of the earth's axis, results in a change in the relative position of the sun as the earth moves in its orbit. This change in the relative position of the sun is reflected in the change that occurs in the angle the sun's rays make with the equatorial plane. This angle is known as the declination angle and is shown schematically in Fig.2.The declination will vary from maximum of $234^{\circ}$ at the summer, Solstice to minimum of $-234^{\circ}$ at the winter. Twice in the year, the declination will be zero, this occurs at spring and autumn Equinox, [3], [4], [5].

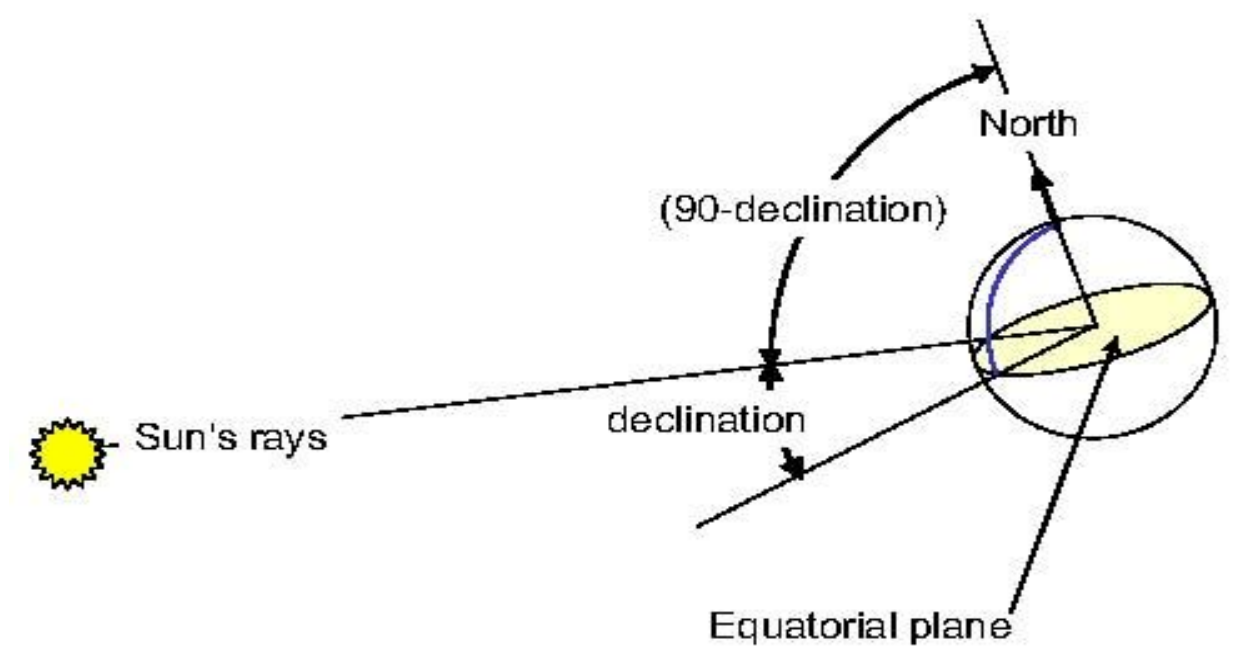

Figure (2) Declination angle .

Mounting the solar panel declination angle to an optimized angle with respect to the horizontal axis must take into consideration the location, the time of the year and the inclined angle of the robot due to the minefield surface and nature. The panel holder mechanism can be oriented in any azimuth range between (South East) (0o) and (South West) (180o) and elevated between vertical (0o) and horizontal (90o) position in preprogrammed steps. Relevant parameters are measured continuously and acquired in the program database and used for panel position adjustments. The mine detection robot moves in the desert through the minefield searching the mines changing its path, direction angles and inclination angle continuously due to the nature of 
the ground of the field wanted to be scanned. Therefore, obvious solution is to fit the solar panel into a movable tracking system installed on the robot that precisely follows the course of the sun along with the robot motion. The designed mechanism and controller is capable of rapidly locking into the sun position and follow the sun during the robot's searching operation.

The controller of the tracking mechanism calculations requires number of variables to produce the net angels of the solar panel that have to be in specified instant like the position of the robot, the daytime, the location of the robot on the earth, and the season of the year. This system is always in synchronization with the rotation of earth, without any extra component, starts at the time of sunrise and goes on as earth rotates on its own axis until the sun set i.e., from 6 AM to 6 PM. Fig. 3 illustrates the synchronization process and the movement of solar panel during the day.

The controller is coded using an algorithm based on astronomical data to produce the final angels of the solar panel, [4], [6], [8]. The panel positioning auto tracking control system is a two axes motorized rotator carrying panel holder composed of PIC18F452 MCU circuit, sensors and a monocrystalline solar panel of 90 watt.

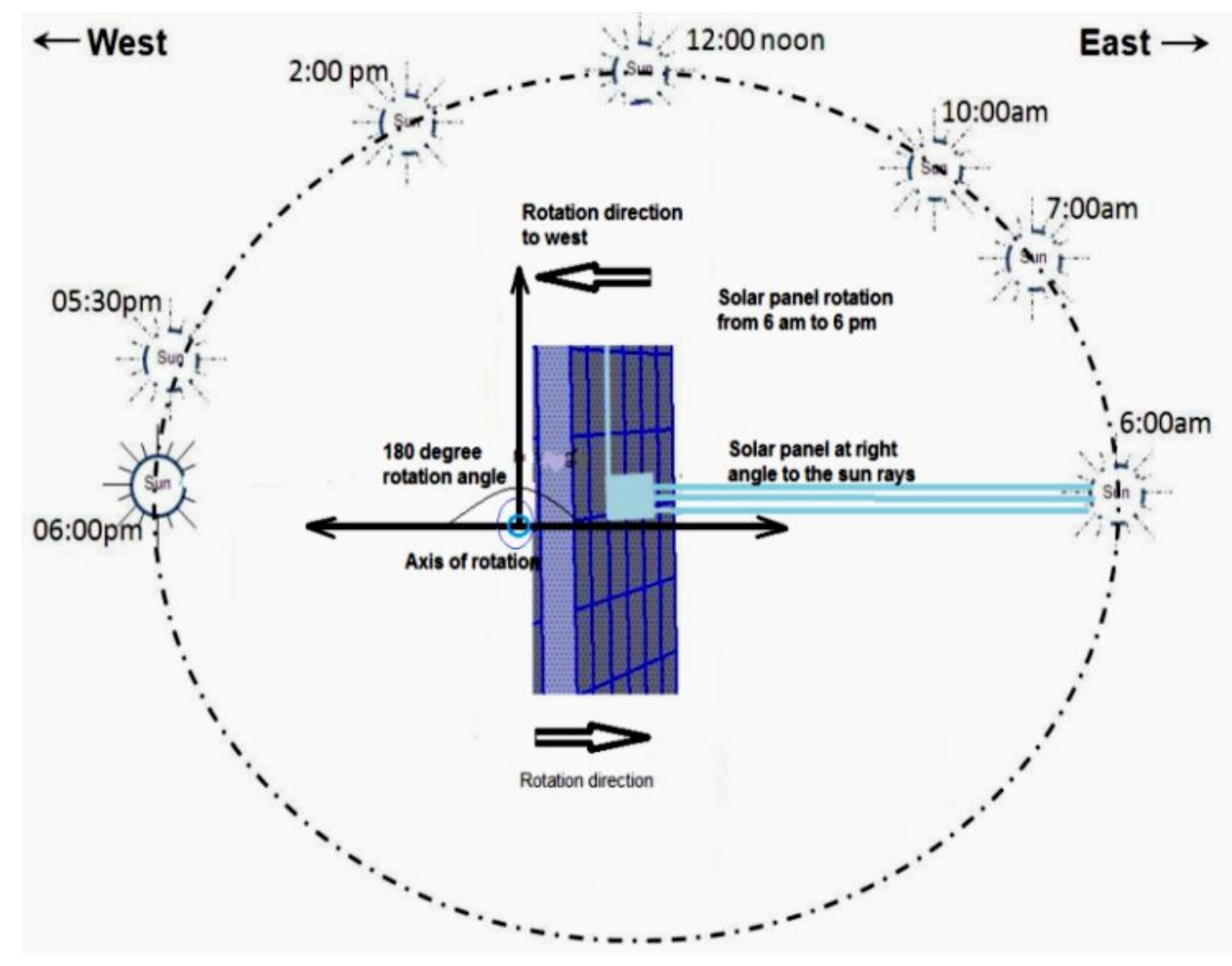

Figure (3) The synchronization process and movement of the panel during the day. The tracker needs to respond quickly to the robot's path changes to avoid energy loss and low charging efficiency. The realized tracking system has a boost converter to raise the output voltage of the solar panel under variable insolation conditions and variable output voltage of the 
panel to a fixed value. The voltage charging process is controlled using microcontroller. The combination of the voltage charging controller and the tracking position is implemented with the use of the algorithm coded on the PIC 18F452 which results in a two axes tracking strategy. This design, along with charge controller boost converter, makes the tracking system complete. The PWM controlled DC-DC boost converter used to deliver power in the lower output voltage range of solar panel, protecting the $24 \mathrm{~V}$ dc battery from overcharged, over discharged condition and overcome the lower output voltage of solar panel in a specified output voltage range. The converter is designed to fix the fluctuating or variable input voltage to a constant output with input range of 10-18.2 volts and for transferring maximum power from the solar PV module to charge the robot's deep cycle batteries then drive motors and electronic circuits. To produce a constant output voltage, a feedback loop is used. The output voltage is compared with a reference voltage and a PWM wave is generated, the microcontroller is used to generate PWM signal to control switching actions.

\section{Operation of the DC-DC converter:}

The scheme shown in Fig. (4) represents the proposed boost converter.

The converter is based on the use of conventional DC -DC converter, operated in continuous conduction mode CCM, connected to solar panel of mono crystalline type, has following parameters :

Maximum power $(\mathrm{P} \max )=90 \mathrm{Watt}$

Voltage at $\mathrm{P} \max (\mathrm{V} \mathrm{mp})=18.2 \mathrm{~V}$

Current at $\mathrm{P} \max (\mathrm{Imp})=4.94 \mathrm{~A}$

Open-Circuit Voltage $(\mathrm{Voc})=22.1 \mathrm{~V}$

Short-Circuit Current (Isc) $=5.35 \mathrm{~A}$

The PV rated voltage is fluctuating between 10and 18.2 Volt DC for variable insolation and it is required to charge $24 \mathrm{~V}$ battery bank. The Boost regulator provides a constant output voltage of 25 volt, which is greater than its input voltage.

Referring to the proposed Boost converter circuit, D2 allows current to flow into the booster but cannot flow out of the battery to a void back feed of power.

The bulky capacitance bank is used to stabilize the PV voltage. L1, switch power BJT S1 and $\mathrm{C} 2$ are the boost regulator components. D2 is used to disconnect the battery if the boost regulator output voltage is less than the battery bank. Two feedback resistances are used for voltage and current sensation.

The boost converter regulator operation is divided into two modes of operation as shown in Fig. 5 ( $\mathrm{a}, \mathrm{b}$, and $\mathrm{c}$ ). The first mode (b), when switch $\mathrm{S}$ is turned ON, the current rises through the inductance $\mathrm{L}$ and the load is supplied thought the energy stored in the capacitor. The second mode (c), when switch S is turned OFF (open), the circuit consists of the solar panel (power source) (PV), inductor and RC load. The energy stored in the inductor during the ON state is discharged to the RC load circuit through the diode D1 and at this point, the input is supplying 


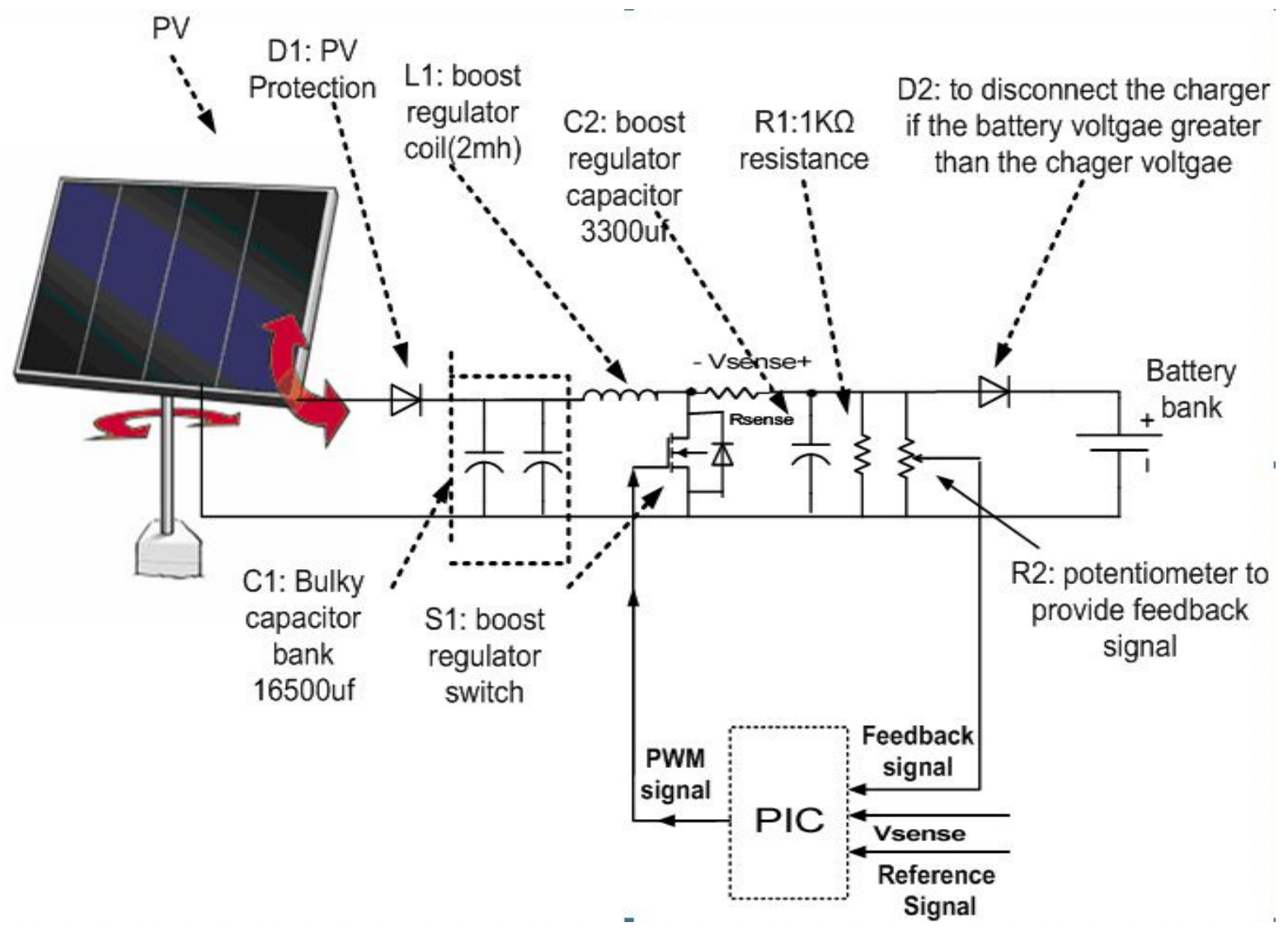

Figure (4) Battery charger boost converter circuit that used in the tracking system.

energy to the inductor. Thus, the inductor current is reducing linearly until the switch $\mathrm{S}$ is turned on again, charging the capacitor at the load side.

Thus, during switch closing time, the inductor gets charged and capacitor is delivering the required power to the load. During switch opening time, the inductor discharges supplying the full power to load and charging the capacitor simultaneously.

When the converter is used in PV applications, the input power, voltage and current change continuously with the atmospheric conditions, thus the converter conduction mode changes since it depends on them. In other word, the average output voltage is controlled by switching on and off time duration. At constant switching frequency, adjusting the on and off duration of the switch is called pulse-width-modulation (PWM) switching. The switching duty cycle, D is defined as the ratio of the on duration to the switching time. In addition, the duty cycle $\mathrm{D}$ is changed continuously in order to boost the varied input value of the PV voltage to a fixed 


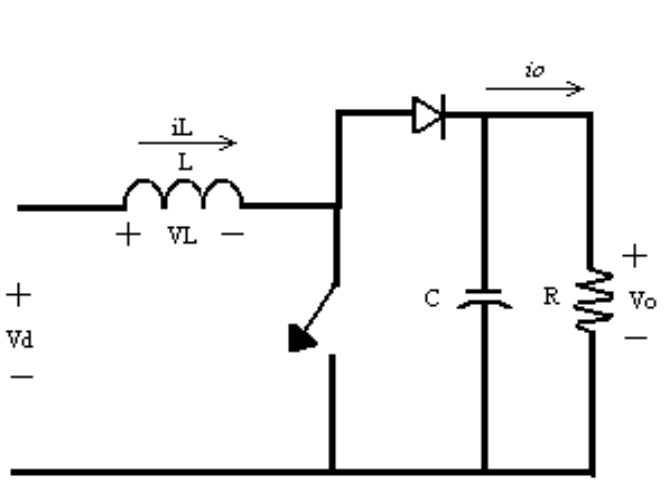

(a)

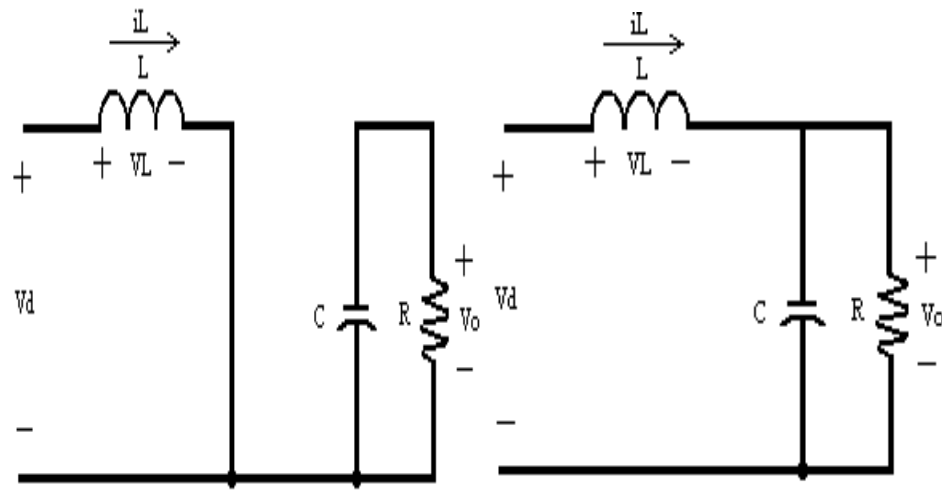

(b)

(c)

(Mode 1 switch on)

(Mode 2 switch off)

Figure (5) (a) Step-up (Boost) dc-dc converter, (b) switch on mode, (c) and switch off mode. output voltage shown in Fig. (6). In order to produce a constant output voltage, current and voltage feedbacks are used. the output voltage will be measured and compared with a reference voltage and the differential value is used to produce a PWM signal. Any changes in the output voltage will lead to the changes of duty cycle in PWM signal. The converter is operated at the switching frequency of $16 \mathrm{KHz}$ the higher the switching frequency, the lower the inductor core size, but the power switch losses are higher, also using a large $\mathrm{L}$ value.

Coil length, $1=8.8 \mathrm{~cm}$

Diameter, $\mathrm{d}=6.4 \mathrm{~cm}$

Inductance value required, $\mathrm{L}=230 \mathrm{H}$

Number of turns, $\mathrm{n}=89$

The peak-to-peak current ripple IL is smaller, requiring lower current rating power switches, but the converter size is increased substantially because a larger inductor core is required. The dc-dc converter operates only in continuous conduction mode CCM, keeping the inductor current flows continuously and never falls to zero as shown in Figure 6 [iL (t) $>0$ ]. When the switch is on and the diode becomes reversed.

In the steady-state analysis we present, the output filter capacitor is assumed to be very large to ensure constant output voltage vo(t) $=\mathrm{Vo}$, when the input voltage to the boost converter is Vd the integral time of the inductor voltage over one period must be zero.

Thus:

Vd ton $+(V d-V o$ toff $=0$

(1)

Dividing both sides by the switching time, Ts, and rearranging terms, we obtain the equation that describes the relationship between input and output voltages, switching time, and duty cycle [6].

$\frac{\text { Vo }}{\text { Vd }}=\frac{\text { Ts }}{\text { Toff }}=\frac{1}{1-D}$

This equation confirms that the output voltage is always higher than the input voltage. Assuming a lossless circuit, $P_{d}=P_{o}$, where $P_{d}$ is the boost input power and $P_{o}$ is the boost output 
power. We have also

$\overline{\text { Io }}=(1-D)$

(3)

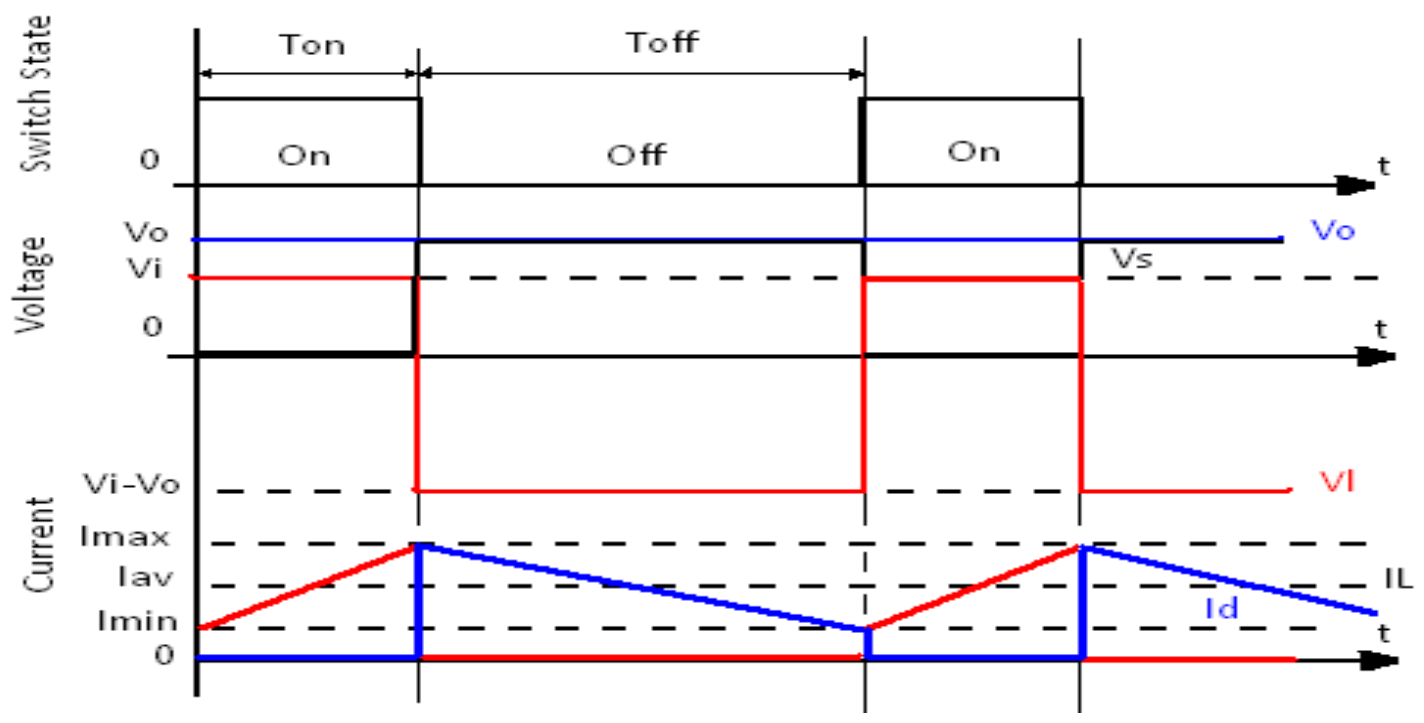

Figure (6) Boost DC-DC converter switching states, and the corresponding voltage and current waveforms .

At the boundary between continuous and discontinuous conduction, $\mathrm{i}_{\mathrm{L}}$ goes to zero at the end of the off interval. At this boundary, the average value of the inductor current is

ILB $=\frac{1}{2}$ iL peak $=\frac{\text { Vd }}{2 L}$ ton $=\frac{\text { TsVo }}{2 L} D(1-D)$

(4)

In converter design the inductor current and the input current are the same $\left(i_{d}=i_{L}\right)$ [7]. Using Eq. (4) we can find that the average output current at the boundary of continuous conduction as:

IOB $=\frac{\text { TsVo }}{2 \mathbf{L}}$ D $(1-\mathrm{D}) 2$

(5)

The output current reaches its maximum when the duty ratio $D=1 / 3=0.333$ :

IOBmax $=\frac{2 \mathrm{Ts} \mathrm{Vo}}{27 \mathrm{~L}}$

(6)

When operating, the average output current at the edge of continuous conduction mode is important, because for a given $\mathrm{D}$, with constant Vo, if the average load current drops below IOB, the current conduction would become discontinuous. In practice, the duty cycle, D, would vary with time in order to keep Vo constant. That common practice allows the tracking of the peak power point. Discontinuous conduction mode is unwanted because it occurs due to a decrease in power and results in a lower inductor current IL since vd is constant, it will be 
completely avoided. The relationship between the input voltage $\mathbf{v d}$, the output voltage Vo, and the duty cycle, $\mathrm{D}$, is given by:

$D=\left(\frac{4 V o}{27 V d}\left(\frac{V o}{V d}-1\right)\left(\frac{I o}{I o B, \max }\right) 1 / 2\right)$

(7)

Vo $=25$ volt Io rated $3 \mathrm{~A}, \mathrm{Vd} 10-18.2$ volt, with variable duty $\mathrm{D}$ from 0.63 to 0.28 .

Since in practice Vo is held constant and D varies in response to the variation in vd , it is more useful to obtain the required duty ratio as a function of load current for various values of the ratio Vo / Vd.

\subsection{Voltage sensor:}

Voltage divider network is used as voltage sensor feedback for the controller. The resistance values chosen are $40 \mathrm{~K}$ and $10 \mathrm{~K}$. This gives the resistance ratio of $10 \mathrm{~K} /(40 \mathrm{~K}+10 \mathrm{~K})=$ 0.2 and therefore gives the maximum input voltage to the $\mathrm{A} / \mathrm{D}$ conversion channel as $0.2 \times 25=5 \mathrm{~V}$.

\subsection{Current Sensor:}

Four resistors network with two parallel paths each having two resistors is used as current sensor for the controller. By connecting a current resistor at the inputs of the two parallel paths, the current from the PV array passing through the resistor and voltage drop across it is calculated by measuring two terminal voltage of it. If the solar panel is assumed operating at its maximum capacity, the maximum output current is $4.85 \mathrm{amp}$, and if $0.5 \mathrm{~W}$ dissipation is allowed for the current sensing resistor, the resistance value can be determined by $\left(0.5=(4.94)^{2} \times \mathrm{R}\right)$ or $\left(\mathrm{R}=0.5 /(4.94)^{2}=0.0205\right)$.

As standard, 0.022 .

\subsection{Charger controller:}

The microcontroller PIC18F452 will attempt to maximize the power input to converter from the solar panel by controlling the duty cycle to keep the solar panel operating at its maximum power point. This is accomplished by continuously adopting voltage and current samples from the panel and using the microcontroller to either increase or decrease the duty cycle of the converter depending on the wattage from the solar panel. The PIC program flowchart according to the following sequence, first the PIC read the PV output voltage and if it is greater than $10 \mathrm{~V}$ DC the PIC calculates duty cycle D, then the PIC start the PWM and wait for $300 \mathrm{mSec}$, then, the PIC read the output voltage and take decision depending upon:

If $\mathrm{V}_{\text {op }}>25$ volt decreases the PWM duty cycle by 1 . 
If $\mathrm{V}_{\text {op }}<25$ volt increases the PWM duty cycle by 1 .

The controller will loop again when the system reaches its steady state operation, the output from the solar panel will oscillate at its highest output value. Charger controller measures always the battery voltage and compares it with the highest and lowest threshold value so that battery is protected from being over charge and over discharge condition. Figure (7) shows the output voltage Vo versus the input voltage Vi. The microcontroller can work on voltage up to $5 \mathrm{~V}$ at its analog to digital port. Therefore, an advantage is the reduction of the cost of using separated A/D converter IC. The analog output of voltage sensor is connected to ADC pins of microcontroller and the A/D conversion is completed by using software program. ADC over the full range of mains voltage $(0 \sim 25 \mathrm{~V})$ scaled from 0 to $5 \mathrm{~V}$. The resolution is $25 / 1024$ or $0.024 \mathrm{~V}$. This is a high resolution.

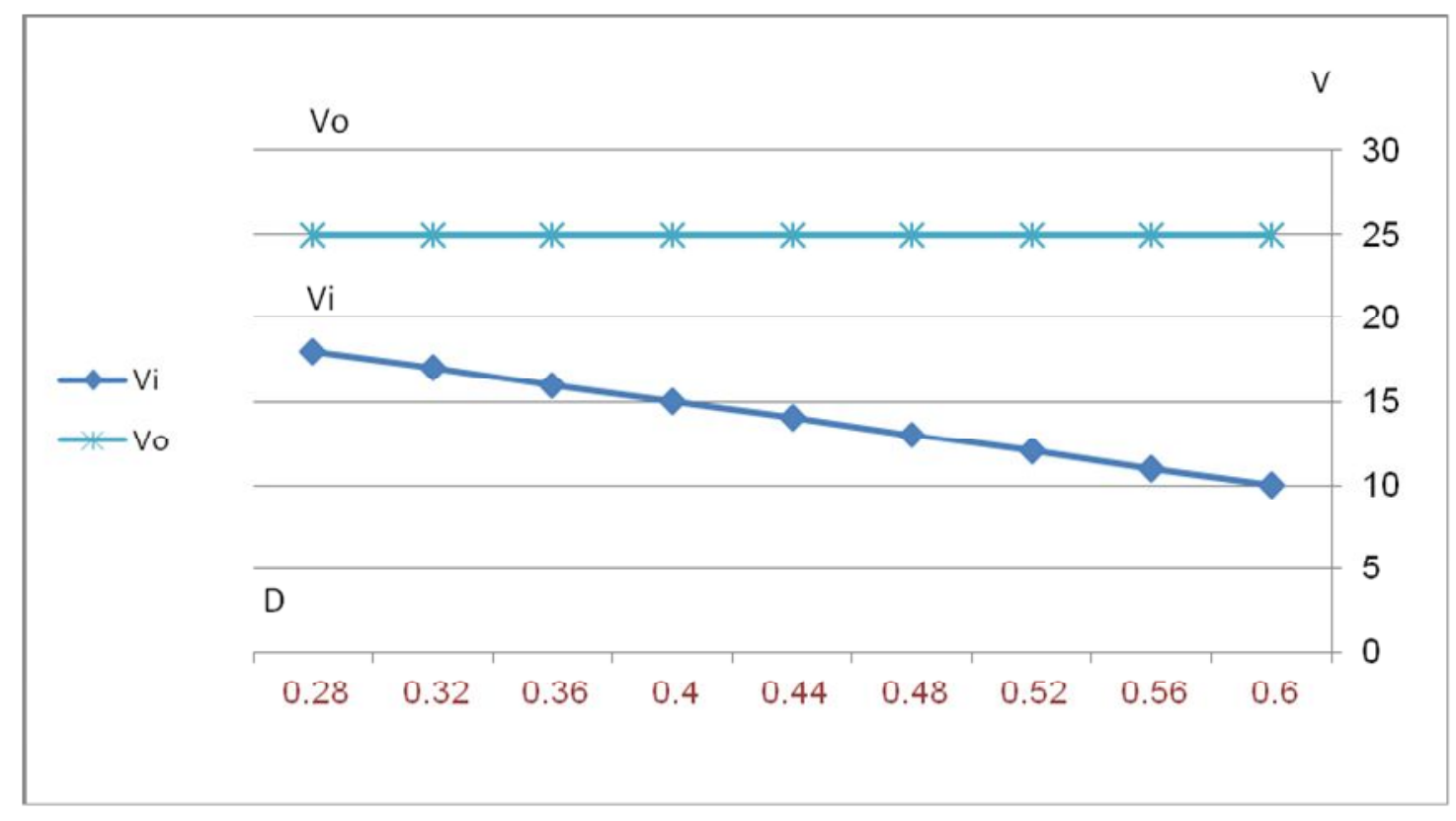

Figure (7) The boost output voltage $\left(\mathrm{V}_{\mathrm{o}}\right)$ versus input voltage $\left(\mathrm{V}_{\mathrm{i}}\right)$ from the panel and duty cycle D for 1 volt step.

\section{Operation of the solar tracking control system and panel positioning device:}

The main idea here is to calculate the difference angles between the robot's path in the field that affect the solar panel net angles and the path of the sun throughout the day. The calculations based on an open loop sensors, which are employed to determine the rotational azimuth, and altitude angle of the tracking axes, guarantees that the solar panel is positioned at the right angles to sunrays. The block diagram of the whole PV panel tracking, positioning and charging device is a schematically shown on Figure (8).

The tilted platform solar panel is hinged at an elevation angle $=\left(90^{\circ}\right.$ - latitude angle $)$ from the 
horizontal rotating plate. This plate rotates about the vertical axis of the robot. The realized sun auto-tracking system of the solar panel consists of the 18F452 MCU, electronic compass, liquid capacitive inclinometer, LCD, 4 relays, electronic processing circuits, photovoltaic modules, two DC motors, two gearboxes 1/40 and the holder mechanism shown in Fig. 10. The azimuth

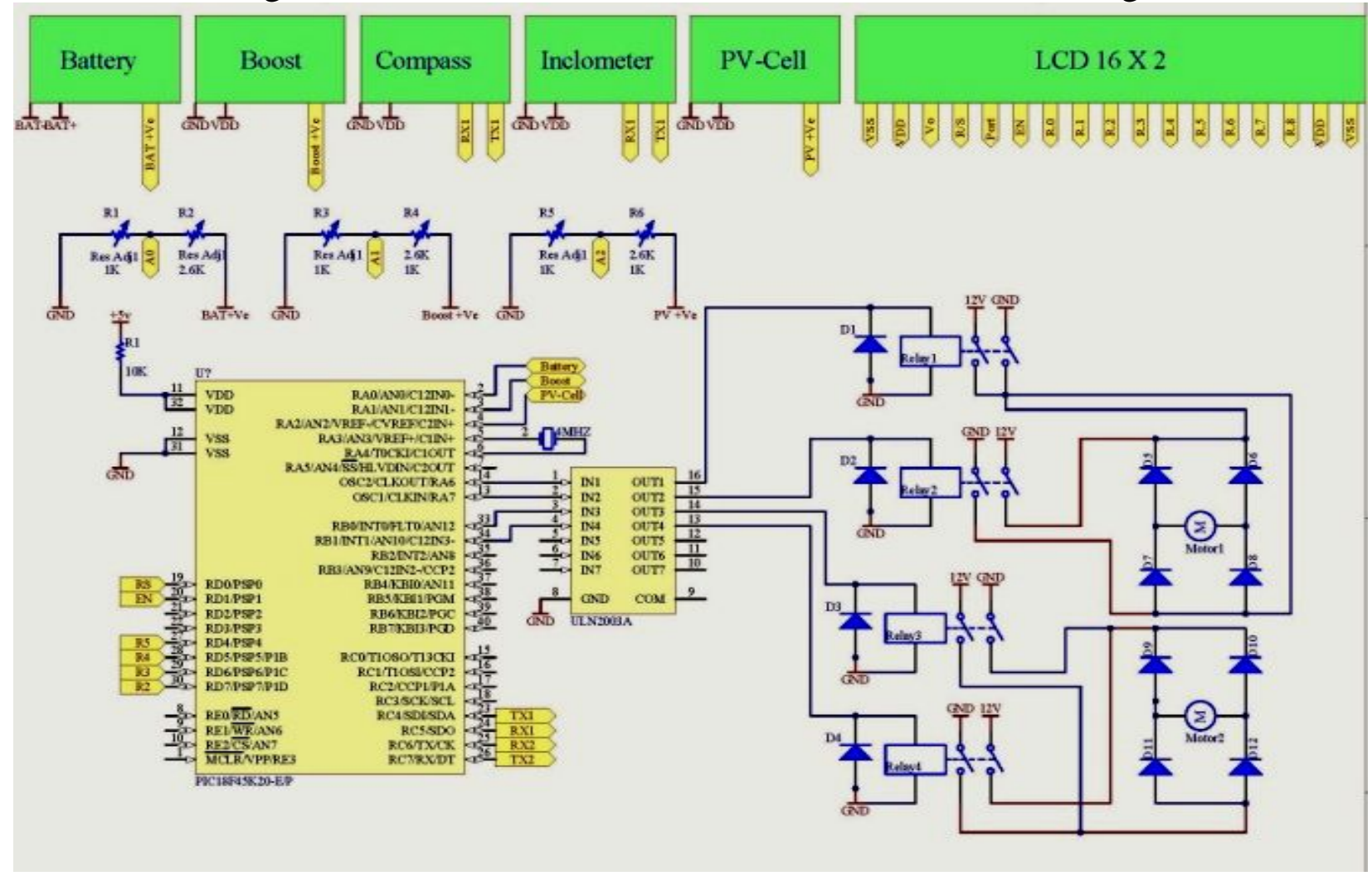

Figure (8) Block diagram of the solar panel tracking and positioning system with charger device.

angle $(\mathrm{Z})$ measurement sensor is electronic compass with tilt and temperature compensation. The tilt angle ( ) measurement sensor is the liquid capacitive based inclinometer used for measuring the net tilt angle of the solar panel with respect to gravity within 0 to $360^{\circ}$ range. This inclinometer features an integrated 16-bit single chip microprocessor, which provides for linearity and temperature compensation. The basic sensor consists of four separate sensing elements whose outputs are combined to provide highly accurate linear output over complete $360^{\circ}$ tilt range. The embedded software virtually eliminates total errors by compensating temperature drifts and sets the sensor dynamics for the ground and the environment at which the mine detection robot moves. The sensor inclinometer is mounted on the vertical mounting plane and connected to the RS232 adapter that is mounted on the horizontal mounting plane. The mechanism designed rotate from $0^{\circ}$ to $180^{\circ}$ angle in a day to track the sun and gives one-half rotation in 12 hours from $6 a m$ to $6 \mathrm{pm}$. It starts its rotation from vertical position at the time of sunrise facing towards southeast (perpendicular to ground) and rotates at the rate of $15^{\circ}$ per 
hour as shown in Fig.3. Due to robot's motion in the field and the ground terrain, the solar panel changes its angles, its azimuth direction angle becomes (Zs) and the inclined angle on the ground ( s) are continuously changed. The microcontroller calculates the difference between the instantaneous change of robot's angel values and the astronomical angel values in the code of the PIC. Microcontroller code calculates the following terms to determine the final position of the solar panel due to sun motion and applies signals to the motors to rotate to achieve the calculated angles.

The Declination angle DE (shown in Fig.2) of the solar panel as a function of the day number is given by:

$\mathrm{DE}={ }^{23.4 \times \operatorname{Sin}\left(\frac{360 \times(284+\mathrm{N})}{365}\right) \text { Degree }}$

(8)

Where $\mathrm{N}$ is the day number of the date for which the declination calculated. January 1st. being day number 1 and expressed as the $\mathrm{N}=$ one, starting with January, thus March 11 would be $\mathrm{N}=31+28+11=70$ and December 31 means $\mathrm{N}=365$.

$\operatorname{Sin}=\cos \mathrm{DE} \times \cos \mathrm{L} \times \cos \mathrm{H}+(\sin \mathrm{DE} \times \sin \mathrm{L})$

L the latitude: is the angle from the equator to a position on Earth's surface (Egypt) [4], [5], [9].

$\mathrm{H}$ : is the angular hour $=15\left(\mathrm{t}_{\mathrm{s}}-12\right)$ (degrees)

It is the angle between the planes of the meridian-containing observer and meridian that touches the earth-sun line. It is zero at solar noon and increases by $15^{\circ}$ every hour (shown in Fig.9). $t_{s}$ : the time for rotation of earth around its own axis in 24 hours .

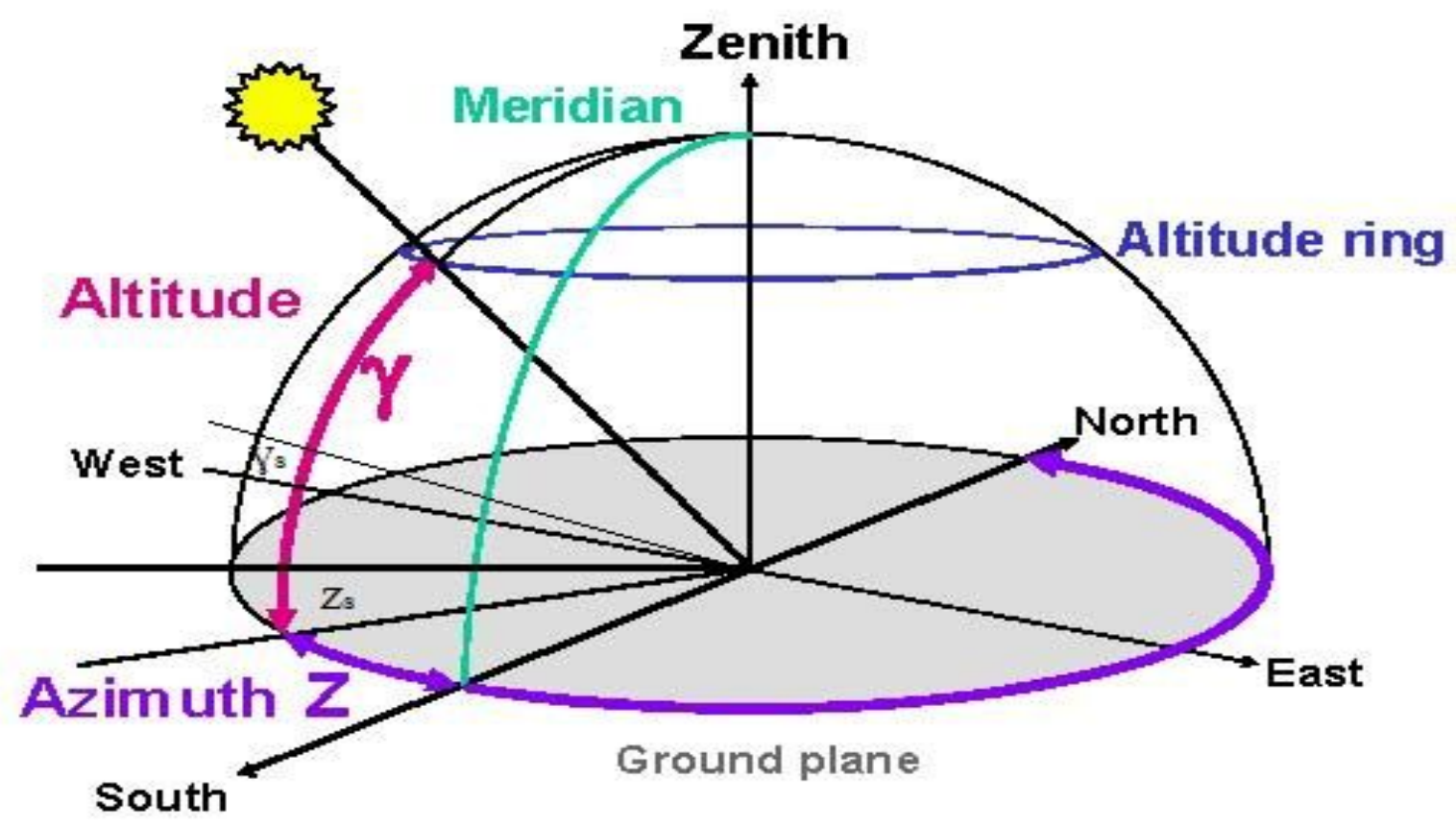


Figure (9) The latitude and azimuth angle of the panel

relative sun angles stand in the northern hemisphere.

The azimuth angle $\mathrm{Z}$ measured by the compass sensor $\mathrm{Z}=\sin ^{-1}\left(\frac{\sin H \times \cos D}{\cos \bigvee}\right)$

(11)

The compass continuously reads the change in azimuth angle of the solar panel (Zs) and the liquid capacitive inclinometer reads the change of the elevation angle of the solar panel ( $s$ ) according to robot's movement in the field. The PIC calculates the difference of angles between the measured angles by the sensors and the desired astronomical programmed solar panel angles $(Z)$ and ( ) to gives (Znet) and ( net) .

$\mathrm{Znet}=\mathrm{Z}-\mathrm{Zs}$

net $=-s$

The signals corresponding to this difference are applied to the motors through relays to rotate in proportion to this difference, changing the angles to become (Znet) and ( net), using programmed movement and measurement in duration of approximately 4 seconds between two steps.

\section{TEST AND RESULTS:}

The hardware structure (shown in Figure 10). The experimental results shows that the proposed design is able to produce a constant $25 \mathrm{~V}$ output voltage with duty cycle of $63 \%$ and $28 \%$, Figure (7). 


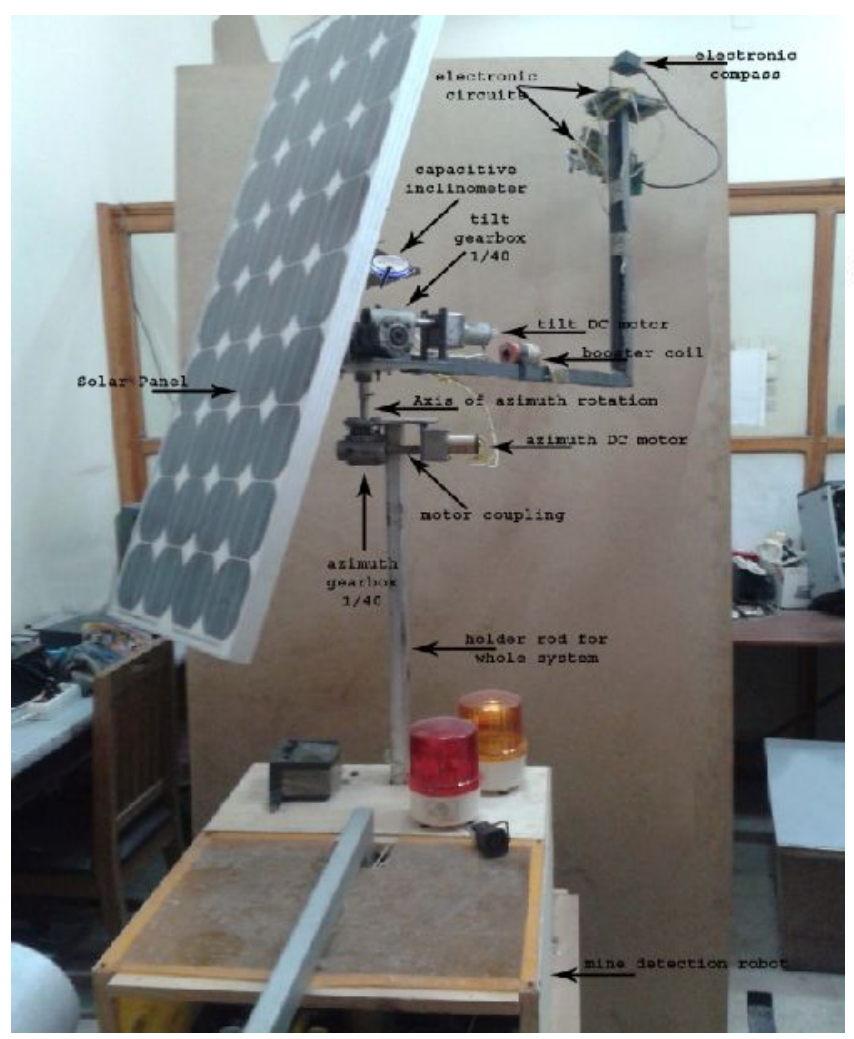

(a)

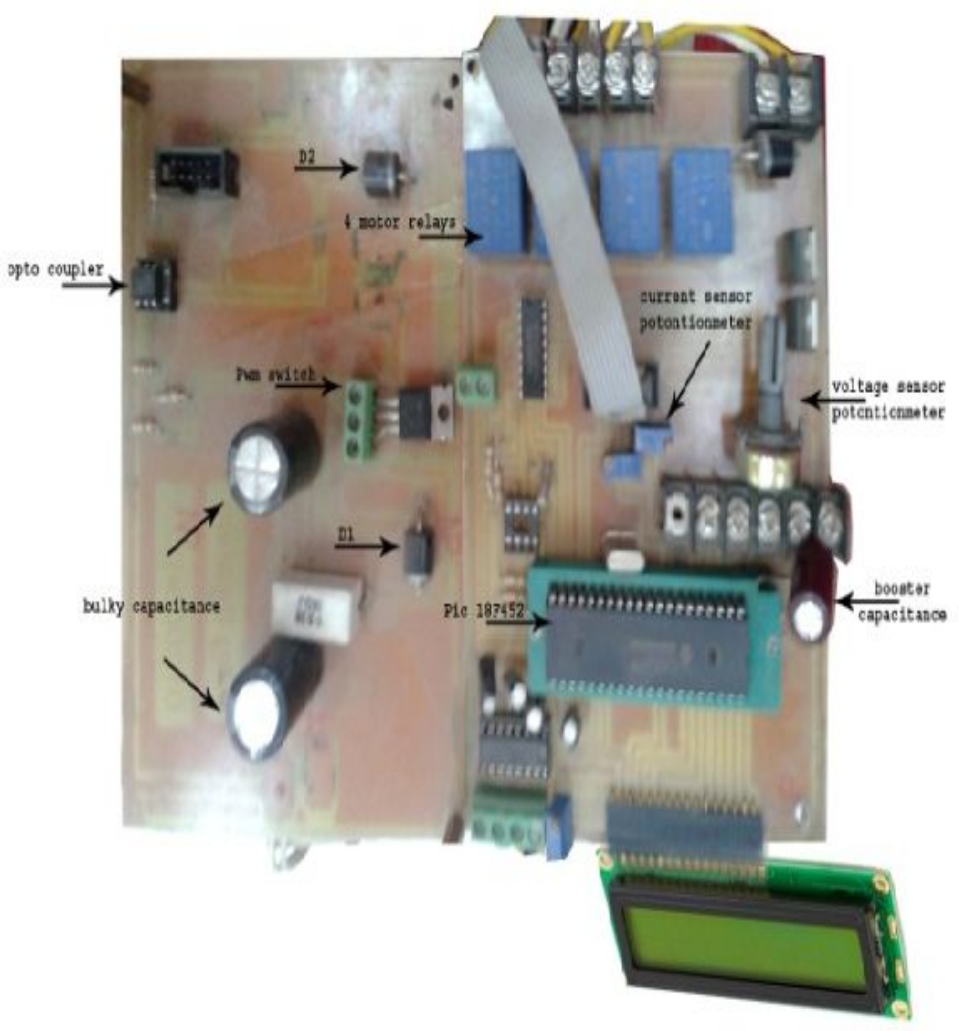

(b)

Figure (10) (a) Practical tracking prototype system (b) Electronic control circuit.

The efficiency and the comparison of the solar panel output power with tracking and without tracking during the robot's motion (shown in Table 1). This Table shows four cases of comparison, as follows:

1- Effect of adjusting fixed angles, at fixed latitude of $30^{\circ}$ in September month and direction $180^{\circ}$.

2- Effect of operating compass sensor, automatic change of the direction only with fixed tilt at $30^{\circ}$.

3- Effect of operating the inclinometer sensor only, automatic change in elevation angle with fixed direction .

4- Effect with completely automated system .

Table (1) Comparison of the output power for the tracking system in four cases . 


\begin{tabular}{|c|c|c|c|c|}
\hline Cases & $\begin{array}{c}\text { Case1 } \\
\text { Fixed }\end{array}$ & $\begin{array}{c}\text { Case2 } \\
\text { compass } \\
\text { sensor only }\end{array}$ & $\begin{array}{c}\text { Case3 } \\
\text { inclinometer sensor } \\
\text { only }\end{array}$ & $\begin{array}{c}\text { Case4 } \\
\text { 2-axis } \\
\text { tracker }\end{array}$ \\
\hline $\begin{array}{c}\text { power } \\
\text { efficiency \% }\end{array}$ & $52.1 \%$ & $89.2 \%$ & $65.7 \%$ & $95 \%$ \\
\hline
\end{tabular}

\section{CONCLUSIONS:}

A novel mechanism of two axes sun tracking system installed on mine detection robot with boost converter is illustrated. A low cost high performance DC-DC closed loop boost converter has been proposed. Design of the complete system has been explained and a prototype has been built and experimentally tested for an input voltage range of 10 to $18 \mathrm{~V}$. For specified input variation, regulated dc output voltage of $25 \mathrm{~V}$ has been obtained resulting in increasing of the efficiency to $95 \%$. The combination of the voltage controller and the tracking position is implemented with the use of the algorithm coded on the PIC 18F452. The design takes into account sun-azimuth-elevation. Tracking system prevents the misalignment of sunrays on the azimuth axis especially because of the change of the robot's direction. The sun-tracking system is also efficient in providing reasonably high precision with much simple design and cost effective. Practically obtained results show significant improvement of the total efficiency of the photovoltaic system in comparison with the fixed one.

\section{REFERENCES:}

[1] M.N.H. Comsan, "solar energy perspectives in Egypt." Proceedings of the fourth Environmental Physics Conference, 10-14 March 2010, Hurghada, Egypt.

[2] Weiping Luo, "A Solar Panels Automatic Tracking System Based on OMRON PLC." Proceedings of the 7th Asian Control Conference, Hong Kong, China, August 27-29, 2009

[3] Kok-Keong Chong, Chee-Woon Wong, "Solar Collectors and Panels, Theory and Applications," Chapter 13, General Formula for On-Axis Sun-Tracking System, book edited by Reccab Manyala, ISBN 978-953-307- 142-8, published: October 5, 2010

[4] Building Environment 1chapter 7 predicting the sun's position, book

[5] SP \& AKI, "Sun and Architecture." GEK 1506 HEAVENLY MATHEMATICS. 
[6] Md. Ismail Hossain, Shakil Ahamed Khan, Md. Shafiullah, "Power Maximization of a Photovoltaic System Using Automatic Solar Panel Tracking along with Boost Converter and Charge Controller." 2012 7th International Conference on Electrical and Computer Engineering 20-22 December, 2012, Dhaka, Bangladesh

[7] Daniel F. Butay Michael T. Miller, "Maximum Peak Power Tracker: A Solar Application." A Major Qualifying Project Report Submitted to the Faculty Of the Worcester Polytechnic Institute April 24th, 2008 pp 001- 127.

[8] Ravi Tejwani \& Chetan S Solanki, " $360^{\circ}$ Sun Tracking with Automated Cleaning System for Solar PV Modules." 2010 IEEE.

[9] A.Ibrahim, M.R.I.Ramadan, A.El-Sebaii, S.M.El-Broullesy, “ Annual Performance of Solar Modules with Tilting Angle Facing South and Sun Tracking in Tanta, Egypt," International Journal of Renew-able Energy Research, IJRER., Vol. 1, No. 1, pp.26-40, 2011. 